\title{
GRB Locations and Spectra with the BALROG
}

\author{
J. Michael Burgess* \\ Max-Planck-Institut für extraterrestrische Physik \\ E-mail: jburgess@mpe.mpg.de

\section{Jochen Greiner} \\ Max-Planck-Institut für extraterrestrische Physik \\ E-mail: jcg@mpe.mpg.de
}

In the era of multi-messenger astronomy, all-sky surveys of transient events serve an important role to provide detection and location information to follow-up instruments. The Fermi-GBM serves as the dominant gamma-ray transient detector with coverage of the entire unocculted sky. We introduce a new method to improve both the precision and accuracy of the Fermi-GBM's ability to locate gamma-ray transients. The method been shown to improve the previously reported systematics of the official localization method. I will discuss the issues with localization, how the method attempts to overcome them, and the still existing problems that must be overcome to provide accurate and precise localizations. These efforts are vital for gravitational wave followup. Additionally, I will discuss the impact of our localization method on observed GRB spectra and argue that we still have a long path to fully understanding the spectra before making accurate physical inferences about them.

7th Fermi Symposium 2017

15-20 October 2017

Garmisch-Partenkirchen, Germany

${ }^{*}$ Speaker. 


\section{Method}

The localization of gamma-ray bursts (GRBs) on the sky has played an important role in the history of understanding these extreme objects. The Burst and Transient Source Experiment ( BATSE) helped to confirm the cosmological origin of GRBs by determining the isotropic sky distribution of over 3000 GRBs [1]. The Fermi Gamma-ray Burst Monitor (GBM), which can monitor the entire sky (with the exception of the portion occulted by the Earth), is currently providing locations for hundreds of GRBs and other transient gamma-ray events every year. With the advent of gravitational wave astronomy and the potential for electromagnetic follow-up of associated events, GBM could play a crucial role in the new era of multi-messenger astronomy.

Using differential rates in each of its 14 non-imaging detectors, GBM is able to provide approximate localization of transient events that can then be used by imaging observatories to search for counterparts at other wavelengths. Unfortunately, the standard GBM localization procedure, the Daughter Of Location (DOL) produces offsets of $8-13^{\circ}$ from the subsequently confirmed true locations for many GRBs localized by GBM [2]. The exact cause of this systematic is unknown and could be attributed to several factors such as imperfections in the simulated spacecraft response and/or model. Here we hypothesize that the systematics in the standard GBM localization algorithm are a byproduct of the assumption that all GRB spectral energy distributions (SEDs) are alike, and of inaccuracies in the detector response matrix ${ }^{1}$ (DRM) modeling. To explore this possibility we have developed a method where the location and spectrum are inferred simultaneously via Bayesian sampling using a new method: the BAyesian Location Reconstruction Of GRBs.

GBM DRMs are built via the General Response Simulation System (GRESS) GEANT4 Monte Carlo code [3] and separated into two components consisting of a direct DRM and an atmospheric scattering DRM of the 14 detectors for each individual GRB position. A grid of direct DRMs created for 272 points on the sky for each of the 12 GBM sodium-iodide (NaI) detectors and 2 bismuth-germinate (BGO) detectors is used as the base response library for DRM generation. These responses exist in an energy compressed format which must be decompressed and added to the atmospheric and satellite-internal scattering response via a series of transformations that depend on GBM's position and orientation with respect to the Earth [5]. The directional dependence of the response adds in an extra complication, as it is too computationally expensive to calculate $R_{d}(\varepsilon, E, \psi)$ for all values of $\psi$ over the sphere, so this operation is undertaken only for the source positions proposed during the posterior sampling process. Therefore, we designed efficient DRM generation software based of the original GBM tool that allows for vectorized and parallel generation of DRMs. The final direct DRM is added to the atmospheric scattering DRM to account for scattering of photons off the Earth's atmosphere [4].

Our full method consists of fitting for both the location and spectrum of simultaneously. This allows for inclusion of the full variability of the posterior into the problem. To accomplish this, We set up the spectral and location dependent likelihood, assign priors, and sample the posterior via Bayesian Monte Carlo. At each iteration, an instrument response for the sampled location is generated and the sampled spectrum is convolved with this response producing a count spectrum.

\footnotetext{
${ }^{1}$ GBM and BATSE DRMs differ from classical instrument DRMs because the effective area and response are combined into one matrix.
} 
This count spectrum is compared with the true data via the proper Poisson profile-likelihood. This process results in a posterior for both the location and spectral parameters.

\section{Results}

We apply this technique to the ten best and worst located GRBs from [2] to assess the viability of the technique. We find in all cases that the technique provides accurate location while not always being precise. In other words, we find that in some cases the error region encapsulates the true location of the source, but can be significantly larger than previously reported. This is indicative that the previously reported error regions are underestimated due to the lack of the inclusion of the full variability of the problem.

Further more, we used Bayesian posterior predictive checks (PPCs) to verify that there was no systematic difference between the best and worst sample. We find that analysis on both samples results in the same quality of fit. Performing a similar analysis on previously reported GBM locations for the same samples shows a severe systematic difference between the samples. Thus, our approach appears to reduce the differences between the samples.

While the improvement in the location analysis in notable, it is important to point out that the improvement was also dependent on the detector selection. Classically, all GBM detectors are utilized to perform location analysis. We found that the inclusion of all detectors resulted in systematics even when using our Bayesian analysis. This is indicative of systematic deficiencies in the GBM instrument response which must be investigated.

\section{Discussion}

The aim of this work is to both understand the causes of the systematic offset in GBM locations from the true location of sources as well as introduce a new technique for location GRBs. We have taken a sub-sample of GRBs that have been previously located by both GBM and imaging telescopes and shown that the tails of the GBM location-offset distribution can be analyzed and corrected. Thus, the technique does not suffer from the systematics of the classical location approach. However, systematics still exist. Properly locating a GRB is dependent on the detector selection and in an as of yet unquantifiable way. We encourage a thorough calibration campaign to understand these issues. Moreover, we are continuing to widen our sample of located GRBs in our own effort to better understand the issues related to locating GRBs and the GBM instrument response.

\section{References}

[1] Michael S Briggs, William S Paciesas, Geoffrey N Pendleton, Charles A Meegan, Gerald J Fishman, John M Horack, Martin N Brock, Chryssa Kouveliotou, Dieter H Hartmann, and Jon Hakkila. BATSE Observations of the Large-Scale Isotropy of Gamma-Ray Bursts. ApJ, 459:40-, March 1996.

[2] V Connaughton et al. Localization of Gamma-Ray Bursts Using the Fermi Gamma-Ray Burst Monitor. ApJS, 216(2):32, February 2015. 
[3] R M Kippen, A S Hoover, M S Wallace, G N Pendleton, C A Meegan, G J Fishman, C A Wilson-Hodge, C Kouveliotou, G G Lichti, A Von Kienlin, H Steinle, R Diehl, J Greiner, R D Preece, V Connaughton, M S Briggs, W S Paciesas, and P N Bhat. Instrument Response Modeling and Simulation for the GLAST Burst Monitor. In THE FIRST GLAST SYMPOSIUM, pages 590-591. AIP, July 2007.

[4] Charles Meegan, Giselher Lichti, P N Bhat, Elisabetta Bissaldi, Michael S Briggs, Valerie Connaughton, Roland Diehl, Gerald Fishman, Jochen Greiner, Andrew S Hoover, Alexander J van der Horst, Andreas von Kienlin, R Marc Kippen, Chryssa Kouveliotou, Sheila McBreen, W S Paciesas, Robert Preece, Helmut Steinle, Mark S Wallace, Robert B Wilson, and Colleen Wilson-Hodge. The Fermi Gamma-ray Burst Monitor. ApJ, 702(1):791-804, September 2009.

[5] W. S. Paciesas, G. N. Pendelton, R. B. Wilson, G. J. Fishman, and C. A. Meegan. Observations of Hard X-ray Continuum Emission from SN1987A. In Bulletin of the American Astronomical Society, volume 20, page 726, March 1988. 